\title{
Lebensstil und gesundheitsrelevantes Verhalten im Jugendalter
}

\section{Von Jürgen Raithel}

\section{Einführung}

Dem gesundheitsrelevanten Verhalten kommt im Jugendalter eine überaus bedeutende Rolle $\mathrm{zu}$, da entsprechende Verhaltensmuster in der Jugendphase habitualisiert und im Erwachsenenalter oft fortgesetzt werden. Besonderes Interesse kommt hierbei den gesundheitsriskanten Verhaltensweisen zu, da diese für einen Großteil der Morbiditäts- und Mortalitätsraten verantwortlich sind (vgl. BZgA 2001; Hurrelmann 2000; Kolip 2000; StBA 2002; Ziegelmann 2002).

Ausgehend von der Gesundheitsdefinition der Weltgesundheitsorganisation aus dem Jahre 1948 (WHO 1948), dass Gesundheit der Zustand des völligen körperlichen, geistigen und sozialen Wohlbefindens und nicht nur das Freisein von Krankheiten und Gebrechen ist, fällt es schwer, das Spektrum gesundheitsrelevanter Verhaltensweisen abzustecken. Mit dem Begriff Gesundheitsverhalten sind nach Kasl und Cobb (1966) alle Verhaltensaspekte gemeint, die im Zusammenhang mit Gesundheit und Krankheitsverhütung stehen. In dieser Perspektive sind unter Gesundheitsverhalten sowohl Verhaltensweisen, die zur Erreichung sowie Erhaltung eines ,positiven“ Gesundheitszustands dienlich sind (eingeschränkter Begriff), als auch Verhaltensweisen, die eine Gesundheitsgefährdung darstellen - also gesundheitsriskantes Verhalten - zu fassen. Da allerdings Gesundheitsverhalten in der eingeschränkten Begriffsbestimmung als volitives krankheitspräventives Verhalten verwendet wird (vgl. Ziegelmann 2002), kommt der Begriff gesundheitsrelevantes Verhalten zu tragen. Dieser Begriff ist von seiner lexikalischen/semantischen Bedeutung eindeutiger und schließt alle Verhaltensklassen, die für den Gesundheitszustand relevant sind, ein. So stellt auch das gesundheitliche Risikoverhalten eine Verhaltensweise der gleichen Verhaltensklasse dar, aber in bipolarer Perspektive (vgl. Schwarzer 1990).

Der Gesundheitsstatus und das gesundheitsrelevante Verhalten der Jugendlichen ist jedoch nicht über die gesamte Kohorte gleich verteilt. Es bestehen vielmehr gesundheitliche Ungleichheiten. Eine Vielzahl von Studien belegen die Existenz gesundheitlicher Unterschiede in der Richtung, dass der Gesundheitszustand in den statusniedrigen Gruppen zumeist erheblich schlechter als in den statushohen Gruppen ist (vgl. zum Überblick Mielck 2000). Die bisherigen Ansätze gesundheitlicher Ungleichheit gehen schwerpunktmäßig von einem Schichtkonzept aus (vgl. Weber 1987; Steinkamp 1993; 1999; Angermeyer/Klusmann 1987; Siegrist 1989; Elkeles/Mielck 1997). Jedoch wird in der soziologischen Diskussion seit mehreren Jahren versucht, die Komplexität der Sozialstruktur detaillierter zu erfassen, als dies mit den traditionellen Konzepten der sozialen Ungleichheit erfolgen kann (vgl. Hradil 1987; 1992; Tomlinson 2003; für die Sozialepidemiologie: Sperlich/Mielck 2003).

Hintergrund hierfür ist die These, dass zum einen die Unterteilung sozialer Strukturen in hierarchisch geordnete Schichten im Kontext grundlegender sozialstruktureller Wandlungen (Wohlstandssteigerung und Wertwandel) an Bedeutung verliert und zum anderen die Heterogenität sozialer Ungleichheit angemessener mit einem Milieu- oder Lebensstilkonzept erfasst werden kann. Eingang in die Diskussion über gesundheitliche Ungleichheit hat bisher vor allem das Lebensstilkonzept gefunden (vgl. Pientka 1994). Abel (1991) geht davon aus, dass Lebensstile die zentrale Schnittstelle zwischen der sozialwissenschaftlichen und der gesundheitswissenschaftlichen Forschung darstellen. Solch ein Ansatz bietet die Möglichkeit einer sehr detaillierten Analyse gesundheitlicher Ungleichheit, in welcher sich die traditio- 
nellen Indikatoren der sozialen Ungleichheit (Bildung, Berufsstatus, Einkommen) gut einordnen lassen (vgl. Abel/Rütten 1994; Hradil 2001).

Betrachtet man die Mortalitäts- und Morbiditätsursachen, so ist festzustellen, dass $50 \%$ der Mortalität der 10 Haupttodesursachen verhaltensbedingt sind (Ziegelmann 2002). D.h., dass die Lebensgewohnheiten resp. Lebensstile einen maßgeblichen Einfluss auf die Mortalität und Morbidität haben (vgl. Klein/Schneider/Löwel 2001; Gerhards/Rössel 2002). So fordert auch Meyer (2001), das Gesundheitsverhalten resp. das gesundheitsrelevante Verhalten anhand eines Lebensstilansatzes zu analysieren. Für die Anwendung eines Lebensstilkonzepts im Zusammenhang mit gesundheitsrelevantem Verhalten Jugendlicher spricht weiterhin die Forderung Franzkowiaks (1986), Risikoverhalten im Lebensweisenkonzept zu thematisieren. Denn das Verständnis des Sinns und der Funktionalität von gesundheitsriskanten Verhaltensweisen wird im Rahmen stresstheoretischer Modelle einseitig auf eine kompensatorische Funktion der Konfliktbewältigung verengt. Damit wird die lebensgeschichtliche Logik ebenfalls auf eine individuelle Bewältigung verschoben, ohne der Einbettung in kulturelle Bedeutungs- und Verhaltensmodelle angemessen Rechnung zu tragen (vgl. Franzkowiak 1986). Ausgehend von dieser Perspektive scheint ein Lebensstilkonzept zur Analyse gesundheitsrelevanten Verhaltens im Jugendalter sehr gewinnbringend (vgl. Raithel 2003b).

Nun stellt sich die Frage, welche Lebensstilkonzeption und damit einhergehende Operationalisierung favoritisiert werden soll. Der Lebensstilbegriff per se hat eine lange Tradition und stammt in der heutigen Lesart von Max Weber (1972). Das Konzept ist allerdings auf dem Umweg über die Vereinigten Staaten zu uns gekommen (vgl. Müller 1992, 371ff). Was nun Lebensstile sind bzw. wie sie definiert sind, ist sehr unterschiedlich. Dies hängt auch damit zusammen, dass zwischen der zunehmenden theoretischen Bedeutung des Lebensstilkonzepts in der Sozialstrukturanalyse und seiner empirischen Erfassung eine Lücke klafft (vgl. Georg 1998). In einer breiten Definition wird unter Lebensstil ein regelmäßig wiederkehrender Gesamtzusammenhang der Verhaltensweisen, Interaktionen, Meinungen, Wissensbestände und bewertenden Einstellungen eines Menschen verstanden (vgl. Hradil 1999, 42).

Als Merkmale der Lebensstile benennt Müller (1992, 377f.) vier Dimensionen: (1) expressives Verhalten, (2) interaktives Verhalten, (3) evaluative Aspekte der Lebensführung und (4) kognitive Aspekte. In einer Metaanalyse formuliert Hartmann (1999) eine Trias bestehend aus Performanz, Mentalität und Strukturkontext, die hier auch leitend sein soll. In der empirischen Lebensstilforschung lassen sich entsprechend den Dimensionen Performanz und Mentalität grob zwei Richtungen unterscheiden (Spellerberg 1996, 76f.):

- Zum einen eine bedürfnis-werttheoretische Lebensstilkonzeption, die Werten, Einstellungen und Lebensplänen eine zentrale Bedeutung für Lebensstile beimisst (vgl. Becker/Nowak 1982; Mitchel 1983; Gluchowski 1987; Richter 1989) und

- zum anderen eine verhaltens- bzw. performanzbezogene Lebensstilkonzeption, die davon ausgeht, dass sich Lebensstile in erster Linie durch ihren Aktivitätsgehalt, sichtbare Verhaltensweisen, expressive Stilisierung und manifeste Alltagsästhetik auszeichnen (vgl. Bourdieu 1982; Lüdtke 1989; Schulze 1992; Spellerberg 1996; Georg 1998).

In Hinsicht auf den Aspekt des Strukturkontextes ist anzumerken, dass ein systematischer Zusammenhang zwischen sozialstruktureller Lage und Lebensstilen von einigen Autoren ausgeschlossen wird; Lebensstile können nach dieser Annahme unabhängig von sozialen Lagen variieren (vgl. Hradil 1987; Richter 1994; Michailow 1994). 
Die vorliegende Untersuchung gründet auf einem Lebensstilansatz, der (expressive und interaktive) Verhaltensvariablen (Performanz) in den Mittelpunkt stellt. ${ }^{1}$ Grundlage hierfür stellen die Arbeiten von Hartmut Lüdtke (1989; 1990, 1992) dar, in welchen die Performanz, also die manifeste Dimension des Verhaltens bzw. der Expressivität, ein neues Gewicht in der Lebensstilforschung erlangt hat. Die Ausrichtung der empirischen Lebensstilanalyse auf Aspekte der Performanz ist jedoch nicht als ein eindimensionales Verständnis zu interpretieren. Lüdtke geht vielmehr von einem elaborierten Lebensstilansatz aus, in dem der Lebensstil als ein Fließgleichgewicht zwischen persönlichen Kompetenzen und Motivationen, der sozioökonomischen Situation und der Performanz-Dimension verstanden wird. Die Aspekte der Kompetenz und Motivation werden als Ergebnisse eines Sozialisationsprozesses gesehen. Zur vertikalen und horizontalen Ungleichheit tragen Lebensstilgruppen insbesondere deshalb bei, weil sie über symbolische und mentale Gemeinsamkeiten zu sozialer und sozialräumlicher Verdichtung führen und über eine selektive Nutzung von Interaktion und sozialen Netzwerken die Träger anderer Lebensstile ausschließen. Kern von Lüdtkes handlungstheoretischem Modell ist die Operationalisierung bewährter Alltagsroutinen. Ausgehend von den theoretischen Grundannahmen des „,constraint-choice-Ansatzes“ beschreibt Lüdtke die Entwicklung eines Lebensstils in einer Sequenz von Wahlhandlungen. Lebensstile entstehen, indem die Akteure ihr Leben bewusst gestalten (vgl. Lüdtke 1989, 53f.). Das konkrete, beobachtbare Verhalten bildet den Kern eines Lebensstils und ist somit ausschließlicher Gegenstand der Lebensstiloperationalisierung.

Im Folgenden wird ein Lebensstilbegriff verwendet, der auf dieser Tradition aufbaut: Lebensstile sind äußerliche, über Verhalten und Symbolgebrauch erkenn- und bestimmbare Gefüge der individuellen Alltagsorganisation. Der Lebensstil gründet sich allerdings nicht nur auf den Gestaltungsmöglichkeiten und Chancen einer Lebenssituation, sondern ist immer auch ein Ergebnis des Umgangs mit Alltagsaufgaben und - jugendspezifischen - entwicklungsbezogenen Anforderungen. Der Lebensstil ist somit die kristallisierte und sich in Routinen verfestigte Struktur einer Suche nach personaler Identität und sozialer Zugehörigkeit. Hiermit stehen die beiden globalen Sozialisationsziele der Individuation und Integration im Zentrum (vgl. Hurrelmann 1994). Lebensstile können so als Ausdruck des Sozialisationsprozesses verstanden werden (vgl. Ulbrich-Herrmann 1998; Dumais 2002).

Ziel der vorliegenden Studie ist es zunächst, in einem ersten Schritt das Spektrum gesundheitsrelevanten Verhaltens Jugendlicher breit zu erfassen und hiernach zu dimensionieren. In einem zweiten Schritt wird eine performanzbasierte Lebensstiltypologie generiert. Drittens soll ermittelt werden, mit welchen Lebensstilen und einzelnen Stilisierungen unterschiedliche gesundheitsrelevante Verhaltensrepertoires assoziiert sind.

\section{Methode}

\subsection{Datenerhebung und Stichprobe}

Datengrundlage der Untersuchung bildet eine Befragung Jugendlicher an weiterführenden Schulen in Bayern, die im Frühjahr 2003 stattfand. Die Auswahl der Schulen und Klassen erfolgte ausschließlich nach alters- und schulformspezifischen Kriterien. Für die Erhebung kam ein standardisiertes Erhebungsinstrument zum schriftlichen Selbstausfüllen im Rahmen von Klassenbefragungen zum Einsatz. Für die Bearbeitung des Fragebogens benötigten die

1) Als die bedeutsamsten Lebensstildimensionen der Typologie für Jugendliche von Georg (1992) stellten sich der Kleidungsstil, Themeninteressen in Printmedien, der Wohnstil und der Musikstil heraus. Hierbei wird deutlich, dass insbesondere die Lebensstilbereiche differenzieren, die expressive Zugehörigkeiten markieren und auf audio-visueller Ebene direkt darstellbar, wahrnehmbar und klassifizierbar sind. 
Jugendlichen in der Regel nicht länger als eine Unterrichtsstunde. Vor der Erhebung wurden die Eltern/Erziehungsberechtigten und Schüler über die Studie informiert und um Mitarbeit gebeten sowie auf die Freiwilligkeit der Teilnahme hingewiesen und Anonymität zugesichert. Geschulte Interviewer führten die Befragung im Klassenverband durch, die nach Vergabe der Fragebögen einige mündliche Instruktionen gaben, die unter anderem die Motivation der Schüler erhöhen sollte, das Antwortverhalten in Richtung sozialer Erwünschtheit, Response Set, Simulations- oder Dissimulationstendenzen zu reduzieren. Um möglichst gegenseitige Beeinflussungen zu vermeiden, erfolgte ein Verweis auf selbständiges Arbeiten. Der Fragebogen enthielt am Ende einige offene Fragen, die für die ,schnelleren" Jugendlichen gedacht waren, um diese zu beschäftigen damit sie nicht die anderen Jugendlichen störten. Die einzelnen Fragebögen waren nach dem Ausfüllen von den Jugendlichen jeweils in ein separates Couvert zu stecken und zu verschließen.

In methodischer Hinsicht ist zu berücksichtigen, dass es bei Selbstauskünften zu Verzerrungen im Sinne sozialer Erwünschtheit kommen kann. Weiterhin können gruppenuntersuchungsspezifische Probleme auftreten. So könnten Befragte durch Einwirken des Gruppendrucks die Annahme und Bearbeitung des Fragebogens nicht ablehnen, wenngleich sie es bei einer anderen Zugangsweise (z.B. postalische Befragung) möglicherweise getan hätten. Eine inadäquate Bearbeitung des Fragebogens kann sich in falschem bzw. nicht-inhaltsorientiertem Antwortverhalten ausdrücken, das gerade bei selbstberichtbasierten Klassenbefragungen mit geringer Verweigerungsquote als vergleichsweise hoch einzuschätzen ist (vgl. Markert 2002).

Insgesamt konnten 663 Jugendliche befragt werden. Ziel der Stichprobenziehung war, eine möglichst schulformspezifisch gleiche Schülerverteilung zu realisieren. Da statistische Zusammenhangsprüfungen auf Grundlage einer möglichst soziallagenspezifisch homogen ermittelten Lebensstiltypologie im Mittelpunkt des Erkenntnisinteresses stand, wurde sich nicht an Repräsentativität orientiert. Der nach unbrauchbaren Fällen und Altersgrenzen bereinigte Datensatz umfasst Aussagen von 608 Schülerinnen und Schülern zwischen 15 und 18 Jahren. In der Analysestichprobe sind 48\% Mädchen und 52\% Jungen vertreten, womit eine sehr homogene Geschlechtsverteilung besteht. Das Durchschnittsalter beträgt 16.3 Jahre $(\mathrm{SD}=1.03)$. Unter den befragten Jugendlichen besuchten $32 \%$ die Hauptschule, $31 \%$ die Realschule und $36 \%$ das Gymnasium.

\subsection{Erhebungsinstrument}

Entsprechend des theoretischen Ansatzes fand die Operationalisierung der Lebensstile über expressiv-ästhetische Verhaltensweisen statt. Es erfolgte eine Differenzierung in 6 Performanzbereiche: Freizeitverhalten, Kleidungsstil, Musikstil, Einrichtungsstil, Filmkonsum und Zeitschriftenkonsum (jeweils 5-stufiges Antwortformat von ,nie“ [1], ,,selten“, ,manchmal“, „oft" bis „,sehr oft" [5]). Als soziodemographische und soziostrukturelle Merkmale galten Alter, Geschlecht und Schulbildung der Jugendlichen sowie die Schulbildung und der Berufsstatus beider Elternteile.

Freizeitverhalten (vgl. Georg 1992). Anhand einer Hauptkomponentenanalyse (VarimaxRotation, Kaiser-Kriterium mit Eigenwert > 1, paarweiser Fallausschluss) wurden 4 Dimensionen ermittelt: Ausgehen (Partys, Kneipe, Disco), kulturelle Aktivitäten (Theater besuchen, klassische Konzerte, Bücher lesen), kreative Aktivitäten (Basteln, Zeichnen, Handarbeit) und Computer-/Internetnutzung (Internet, Computer spielen/arbeiten). Mit der VierFaktoren-Lösung ließen sich 63.1\% der Varianz der Ausgangsvariablen erklären (Cronbachs Alpha liegt zwischen .57 und .66).

Film- und Fernsehkonsum (Video/DVD, Kino, Fernsehen) (vgl. Ulbrich-Herrmann 1998, 113f.; Georg 1992; Fischer 1992, 223). Mittels Hauptkomponentenanalyse konnten 3 Dimensionen bestimmt werden. Mit den Dimensionen Actionformat (Action-/Katastrophenfil- 
me, Kriegsfilme, Kung-Fu-/Karate-/Boxfilme, Science-Fiction, Horrorfilme), Informationsformat (Dokumentarfilme, Nachrichten, Politische Sendungen) und Unterhaltungsformat (Unterhaltungs-/Talkshows, Soaps, Musikvideos) werden 56\% der Gesamtvarianz erklärt (Cronbachs Alpha liegt zwischen .53 und .78).

Musikkonsum (vgl. Ulbrich-Herrmann 1998, 110f.; Georg 1992; 1998, 149; Fischer 1992, 224). Die Hauptkomponentenanalyse der Musikvorlieben führte zur Bildung von 3 Dimensionen. Mit den Dimensionen moderne Tanzmusik (Techno, Discomusik), provokative Jugendmusik (Punk, Independent, Heavy Metal) und anspruchsvolle Musik (Jazz, Klassik) können 67.4\% der Gesamtvarianz erklärt werden (Cronbachs Alpha liegt zwischen .50 und .76).

Kleidungsstil (vgl. Ulbrich-Herrmann 1998, 105f.; Georg 1992; 1998, 152; Fischer 1992, 225). Es lassen sich drei Skalen ermitteln. Die gefundene dreifaktorielle Lösung mit den Dimensionen hip/flippig (verrückt, frech, ausgefallen), qualitätsbewusst/markenorientiert (korrekt, qualitätsbewusst, markenorientiert, elegant) und salopp (zeitlos, zurückhaltend, praktisch) erklärt 58.4\% der Varianz (Cronbachs Alpha liegen zwischen .55 und .76).

Zeitschriftenleseverhalten (vgl. Ulbrich-Herrmann 1998, 116f.; Georg 1998, 149f.). Mit den fünf Dimensionen der Lesepräferenzen Zeitgeschehen (,Spiegel“, „Focus“, Tageszeitung), „Männerthematik“ (Pornographische Zeitschrift, „Playboy“, „P.M.“), Fachzeitschriften (Autozeitschrift, Sportzeitschrift, PC-Zeitschrift), Jugendzeitschriften (,Bravo“, „Mädchen“) und Unterhaltungsmagazine (Kinomagazin, Musikmagazin) können $68.8 \%$ Gesamtvarianz erklärt werden. Die Werte für Cronbachs Alpha sind mit .57 bis .77 ausreichend bis gut.

Einrichtungsstil (vgl. Ulbrich-Herrmann 1998, 105f.; Georg 1992). Die gefundene dreifaktorielle Lösung mit den Dimensionen ordentlich (sauber, sachlich, stilvoll), kreativ (phantasievoll, flippig) und bequem (gemütlich, praktisch, komfortabel) erklärt 64.9\% der Varianz (Cronbachs Alpha liegt zwischen .61 und .72).

Das operationalisierte Spektrum des gesundheitsrelevanten Verhaltens erstreckt sich über 7 unterschiedliche Verhaltensbereiche. Dies sind: Substanzkonsum, Ernährungsverhalten, Bewegungsverhalten (Sportaktivität), Zahnpflegeverhalten, Sexualverhalten, sonnenbezogenes Verhalten und explizit risiko-konnotative Aktivitäten (jeweils 5-stufiges Antwortformat von ,nie“ [1], ,selten“, ,,manchmal“, ,oft“ bis ,,sehr oft" [5]).

Substanzkonsum (12-Monats-Prävalenz) (vgl. Kolip 1997a). Anhand der Hauptkomponentenanalyse konnten vier Dimensionen ermittelt werden. Mit den Dimensionen illegale Drogen (Schnüffeln, Heroin), Alkoholkonsum (Wein, Schnaps), Rauchen (Zigaretten, Cannabis) und Medikamentenkonsum (ohne Pille) können insgesamt 77.5\% der Varianz der Ausgangsvariablen aufgeklärt werden (Cronbachs Alpha = .55).

Ernährungsverhalten (vgl. Kolip 1995; Raithel 2002). Hauptkomponentenanalytisch ließen sich die Dimensionen nährstoffbezogen hochwertiger Lebensmittel (Frisches Obst, Jogurt/Quark, Gemüse, Vollkornbrot, Müsli/Frühstücksflocken), hochkalorisch, nährstoff-ungünstige Nahrungsmittel ((Kartoffel-)Chips/Nüsse, Süßigkeiten, Kuchen/Gebäck/Plätzchen, weißes Brot/Brötchen) sowie proteinreiche nährstoffbezogen unausgewogene Lebensmittel (Fleisch, Wurst, Fast-Food) bestimmen. Die drei Dimensionen klären insgesamt 65.2\% der Varianz der Ausgangsvariablen auf (Cronbachs Alpha = .55).

Explizit risiko-konnotative Aktivitäten (vgl. Raithel 2001; 2003a). Das Instrument umfasst die Dimensionen waghalsige Aktivitäten (Straßenkreuzung blind überqueren, Strommastklettern, S-/U-Bahn-Surfen, Gleisroulette) und Risk-Fashion Aktivitäten (Fallschirmspringen, Drachen-/Gleitschirmfliegen, Bungee-jumping, in Sprungtuch fallen). Beide Dimensionen erklären $67.7 \%$ der Varianz (Cronbachs Alpha $=.55$ ). 
Zahnpflegeverhalten wurde über die Frage „Wie häufig putzt Du Dir Deine Zähne?“ (,nach jeder Mahlzeit“, „,2-mal täglich“, „1-mal täglich“, ,,jeden zweiten Tag“, ,seltener“) fünfstufig erhoben.

Sonnenschutzverhalten. Die Erfassung des Sonnenschutzverhaltens fand über die offene Frage „Wie viele Sonnenbrände hattest Du im gesamten letzten Jahr (auch beim Wintersport oder Solarium)?" statt. Für die weitere Analyse erfolgte eine Transformation der absoluten Zahlen in einen Wertebereich zwischen 1 und 5.

Musiklautstärkekonsum („Hörst Du gerne laute Musik (Disco..)?“) und schützendes Sexualverhalten gegenüber ungewollter Schwangerschaft und AIDS wurden jeweils über eine fünfstufige Häufigkeitsskala von ,nie“ bis ,immer“ operationalisiert.

Bewegungsverhalten. In das Konzept floss die Operationalisierung des Sportaktivitätsniveaus (vgl. Kurz et al. 1996) und die (sitzende) Aufenthaltsdauer vor dem Computer, Videospiel und/oder Fernseher gleichfalls mittels fünfstufiger Antwortskala ein. Diese Items bilden einen Summenindex.

\section{Ergebnisse}

\subsection{Dimensionen gesundheitsrelevanten Verhaltens}

Die Identifikation der dimensionalen Struktur gesundheitsrelevanter Verhaltensweisen erfolgte anhand einer Hauptkomponentenanalyse mit Varimax-Rotation (paarweiser Fallausschluss). Nach dem Scree-Test ergab sich eine Fünf-Faktoren-Lösung (Eigenwerte: 2.6; 1.5; $1.3 ; 1.1 ; 1.0$ ), die insgesamt $56.7 \%$ der Varianz aufklärt (s. Tab. 1).

Tabelle 1: Gesundheitsrelevante Verhaltensweisen (rotierte Komponentenmatrix)

\begin{tabular}{|l|r|r|r|r|r|c|}
\hline & $\mathbf{I}$ & $\mathbf{I I}$ & $\mathbf{I I I}$ & $\mathbf{I V}$ & $\mathbf{V}$ & $\mathbf{h}^{\mathbf{2}}$ \\
\hline Illegale Drogen & $\mathbf{. 6 5}$ & -.08 & .18 & -.05 & -.03 & .46 \\
\hline Sonnenbrände & $\mathbf{. 5 2}$ & .15 & .11 & .03 & .11 & .32 \\
\hline Sexualschutzverhalten & $\mathbf{. . 5 0}$ & .24 & -.10 & -.14 & .12 & .35 \\
\hline Bewegungsverhalten & .07 & $\mathbf{. 7 4}$ & -.24 & .12 & -.09 & .64 \\
\hline Zahnhygiene & -.24 & $\mathbf{. 7 3}$ & .10 & -.01 & -.14 & .62 \\
\hline Hochwertige Ernährung & .07 & $\mathbf{. 6 4}$ & -.09 & -.29 & .27 & .58 \\
\hline Laute Musik & -.01 & -.02 & $\mathbf{. 6 8}$ & .40 & -.19 & .66 \\
\hline Cannabis-/Tabakkonsum & .21 & -.09 & $\mathbf{. 6 7}$ & .08 & .14 & .53 \\
\hline Alkoholkonsum & .31 & -.09 & $\mathbf{. 6 6}$ & -.13 & .08 & .57 \\
\hline Risk-Fashion & -.03 & -.01 & .13 & $\mathbf{. 8 2}$ & .10 & .71 \\
\hline Waghalsigkeit & .37 & -.06 & -.01 & $\mathbf{. 6 6}$ & -.05 & .67 \\
\hline Medikamentenkonsum & -.16 & -.09 & .01 & .05 & $\mathbf{. 8 3}$ & .72 \\
\hline Hochkalor. Ernährung & .34 & .07 & .12 & .03 & $\mathbf{. 5 8}$ & .56 \\
\hline
\end{tabular}

Es lassen sich fünf Dimensionen des gesundheitsrelevanten Verhaltens Jugendlicher identifizieren:

- Exponiertes Risikoverhalten (12.3\% erklärte Varianz)

- Proaktives Gesundheitsverhalten (12.3\% erklärte Varianz)

- Gesundheitsriskantes Sozialkontaktverhalten (11.7\% erklärte Varianz)

- Explizit risiko-konnotative Verhaltensweisen (10.9\% erklärte Varianz)

- Verdecktes gesundheitliches Risikoverhalten (9.5\% erklärte Varianz) 
Die extrahierten Faktoren korrelieren mit -.01 bis .28 nur geringfügig untereinander, weshalb von relativ unabhängigen Dimensionen gesprochen werden kann. Die extrahierte Faktorenstruktur weist intern recht homogene gesundheitsförderliche bzw. gesundheitsabträgliche Verhaltensrepertoires auf, die interdimensional gut diskriminieren.

Der Faktor I (Exponiertes Risikoverhalten) ist durch den Konsum von Schnüffelstoffen und Heroin, der Sonnenexposition und dem Sexualverhalten gekennzeichnet. Hierbei handelt es sich um Verhaltensweisen mit einem exponierten Risiko. Die höchste Faktorenladung besteht für den Drogenkonsum, gefolgt von den Sonnenbränden. Das schützende Sexualverhalten lädt negativ, worin sich die gesundheitsriskante Ausrichtung des Faktors widerspiegelt.

Der zweite Faktor (proaktives Gesundheitsverhalten) wird durch Bewegungsaktivität (Summenindex aus Sportaktivität und recodiertem Bewegungsmangel), Zahnpflege und einer ernährungsphysiologisch hochwertigen Lebensmittelauswahl bestimmt. Diese proaktiven Verhaltensweisen können aufgrund ihrer gesundheitsförderlichen Gerichtetheit im Sinne eines volitiven Verhaltens verstanden werden (vgl. Allmer 1990).

Faktor III (gesundheitsriskantes Sozialkontaktverhalten) ist durch solche gesundheitsriskanten Verhaltensweisen charakterisiert, die vor allem im sozialen Kontext von Bedeutung sind und eine sozialintegrierende Funktion übernehmen. Nahezu gleich stark laden auf diesem Faktor das laute Musikhören, der Konsum von Cannabis und Tabak sowie der Alkoholkonsum. Die relativ höchste Nebenladung findet sich für den Musiklautstärkekonsum, und zwar mit den explizit risiko-konnotativen Verhaltensweisen.

Der Faktor IV (explizit risiko-konnotative Verhaltensweisen) wird durch die waghalsigen und Risk-Fashion-Aktivitäten gekennzeichnet, wobei die Risk-Fashion-Aktivitäten höher laden. Mit dieser Dimension wird die „Exklusivität“ des explizit risiko-konnotativen Verhaltens im interdimensionalen Vergleich herausgestellt. Für die waghalsigen Aktivitäten besteht dennoch eine höhere Nebenladung mit dem exponierten Risikoverhalten.

Der letzte Faktor (verdecktes gesundheitliches Risikoverhalten) ist durch Medikamentenkonsum und eine hochkalorische, ernährungsphysiologisch ungünstige Lebensmittelauswahl charakterisiert. Der Medikamentenkonsum hat hierbei die höhere Faktorenladung. Beiden Verhaltensweisen ist gemein, dass sie ein verdecktes bzw. unauffälliges Risikoverhalten darstellen.

Die Häufigkeitsverteilungen sowie Mittelwerte und Standardabweichungen für die einzelnen gesundheitsrelevanten Verhaltensdimensionen sind Tabelle $2 \mathrm{zu}$ entnehmen. Die Bildung der Indizes erfolgte über den Mittelwert. Für die Dimension exponiertes Risikoverhalten wurde das Sexualschutzverhalten als „negatives Sexualschutzverhalten“ recodiert, um die gleiche Polarisierung und inhaltliche Ausrichtung mit der Sonnenexposition und dem Konsum illegaler Drogen zu erreichen. 
Tabelle 2: Häufigkeiten und statistische Kennwerte der gesundheitsrelevanten Verhaltensdimensionen (Angaben in Prozent)

\begin{tabular}{|l|c|c|c|c|c|}
\hline & Fak. I & Fak. II & Fak. III & Fak. IV & Fak. V \\
\hline nicht - bzw. wenig zutreffend & 89.6 & 11.2 & 50.8 & 78.0 & 61.2 \\
teils/teils & 8.7 & 40.2 & 33.1 & 15.5 & 26.2 \\
zutreffend bzw. voll zutreffend & 1.6 & 48.6 & 16.1 & 6.6 & 12.6 \\
\hline Mittelwert & 1.4 & 3.4 & 2.5 & 1.9 & 2.4 \\
Standardabweichung & 0.7 & 0.8 & 1.0 & 0.9 & 1.0 \\
\hline Mittelwert Mädchen & 1.3 & 3.6 & 2.2 & 1.7 & 2.5 \\
Mittelwert Jungen & 1.6 & 3.2 & 2.9 & 2.1 & 2.4 \\
T(T-Test) & $5.1 * *$ & $-6.1 * *$ & $8.9 * *$ & $6.0^{* *}$ & -1.4 \\
\hline
\end{tabular}

Mittelwerte: $1=$ nicht zutreffend; 5 = voll zutreffend $\mathrm{p}=* *<.001$

Für das exponierte Risikoverhalten (Fak. I) (Konsum illegaler Drogen, Sonnenexposition, negatives Sexualschutzverhalten) zeigt sich, dass dieses Verhalten unter den befragten Jugendlichen am seltensten praktiziert wird. Rund $90 \%$ gaben an, sich überhaupt nicht oder nur in einem sehr geringen Ausmaß so zu verhalten. Hingegen erhielt das proaktive Gesundheitsverhalten (Fak. II) (Bewegungsverhalten, Zahnhygiene, hochwertige Ernährung) die höchste Zustimmung. Knapp die Hälfte der Jugendlichen verhalten sich gesundheitsförderlich, indem sie Sport treiben, ihre Zähne täglich putzen und sich hauptsächlich von ernährungsphysiologisch hochwertigen Lebensmitteln ernähren. Das hier benannte gesundheitsriskante Sozialkontaktverhalten (Fak. III) (lautes Musikhören, Cannabis-/Tabakkonsum, Alkoholkonsum) wird innerhalb der gesundheitsriskanten Verhaltensweisen von den Jugendlichen noch am meisten ausgeführt. Jeder sechste Jugendliche zeigt solch ein Verhalten. Jedoch ist immerhin die Hälfte der Jugendlichen abstinent bzw. im Konsum sehr zurückhaltend. Die explizit risiko-konnotativen Verhaltensweisen (Fak. IV) (Risk-Fashion Aktivitäten, waghalsige Aktivitäten) sind zusammen mit dem exponierten Risikoverhalten die am wenigsten ausgeübten gesundheitsriskanten Verhaltensweisen. Über drei Viertel der Jugendlichen gaben an, waghalsige oder Risk-Fashion Aktivitäten nicht auszuführen und auch keine Affinität dazu zu haben. Das verdeckte gesundheitliche Risikoverhalten (Fak. V) (Medikamentenkonsum, hochkalorische Ernährung) ist wiederum häufiger anzufinden und ähnelt in der Häufigkeitsverteilung dem gesundheitsriskanten Sozialkontaktverhalten. Fast jeder achte Jugendliche nimmt oft Medikamente und verzehrt häufig ernährungsphysiologisch ungünstige, hochkalorische Lebensmittel.

Im Geschlechtervergleich zeigt sich, dass Jungen häufiger gesundheitsriskante Verhaltensweisen ausüben als Mädchen (vgl. Kolip 1997a). Die stärksten Differenzen bestehen im gesundheitsriskanten Sozialkontaktverhalten zuungunsten der Jungen: diese trinken und rauchen öfter und hören häufiger laute Musik. Dieser Befund widerspricht allerdings den Ergebnissen, dass sich Jungen und Mädchen in der Quantität des Tabak- und Alkoholkonsums angeglichen hätten (vgl. Kolip 1997b; Sieverding 2000). Dies mag möglicherweise mit dem jungen Alter der hier befragten Jugendlichen und auch mit einem Regionalitätseffekt (hier ländliches Bayern) zusammenhängen. Erwartungsgemäß dominieren die Mädchen im proaktiven Gesundheitsverhalten. Das verdeckte gesundheitliche Risikoverhalten wird in einem sehr geringen Ausmaß (nicht statistisch signifikant) ebenfalls von den weiblichen Jugendlichen öfter praktiziert. Ein getrennt geschlechtsspezifischer Mittelwertvergleich für den Medikamentenkonsum und die hochkalorische Ernährung zeigt, dass die Mädchen signifi- 
kant häufiger Medikamente einnehmen (vgl. Kolip 1997a), während die Jungen tendenziell mehr ernährungsphysiologisch ungünstige, hochkalorische Lebensmittel zu sich nehmen.

\subsection{Die ermittelten Lebensstile}

Für die Erstellung der Lebensstiltypologie kam die Clusteranalyse zum Einsatz. Die Interkorrelationen schwanken zwischen $r=.00$ und $r=.52$ und liegen damit unterhalb des vorgeschlagenen Grenzwertes ( $r>.90$, Backhaus et al. 1990, 155), so dass alle 21 Variablen (Summenscores) in die Clusteranalyse eingehen können. Sämtliche Variablen wurden vor der Clusteranalyse z-transformiert, um eine Vergleichbarkeit mit Clusterlösungen anderer Studien zu ermöglichen. Die Bestimmung der Clusterzahl erfolgte zunächst über das hierarchische, agglomerative Verfahren von Ward (1963). Die Analyse ergab, dass sich die Fälle in 4 Cluster gruppieren lassen. Im zweiten Schritt erfolgte die Bildung der Lebensstiltypologie mit einer k-means Clusteranalyse (mit dem Verfahren Quick-Cluster innerhalb des Statistikprogramms SPSS 11.0) unter Zuhilfenahme der Vorinformation über die mögliche Clusterzahl qua Ward-Methode als Anfangspartition.

Ausgehend von dem Erkenntnisinteresse, homogene und voneinander deutlich zu unterscheidende Lebensstiltypen zu ermitteln, ist die Auswahl der definitiven Clusterzahl ein schwieriges Unterfangen, weil hierfür keine theoretischen Vorentscheidungen existieren. Es gilt somit einen erkenntnistheoretischen Kompromiss zwischen einer Grobstruktur bzw. Einfachstruktur und einer Feinanalyse zu treffen (vgl. Georg 1998, 162). In der Grobstruktur gehen spezielle Ausprägungen verloren und in einer Feinanalyse können einzelne Typen überzeichnet werden. Aus inhaltlich-interpretatorischen Kriterien fiel die Entscheidung auf eine 4-Cluster-Lösung, die sich ebenfalls nach formal-statistischen Entscheidungskriterien für die Bestimmung der Clusterzahl anhand der erklärten Varianz (Eta-Quadrat), deren Differenz zwischen zwei Lösungen (Delta-Eta-Quadrat) sowie der Pseudo-F-Statistik konsolidierte (vgl. Bacher 1996, 316f.).

Die clusteranalytisch ermittelte Lebensstiltypologie und die ihr grundlegenden expressivästhetischen Stilelementausprägungen sind Tabelle 3 zu entnehmen. Als den Lebensstil charakterisierend gelten über- und unterdurchschnittliche Werte im Verhalten der Jugendlichen eines bestimmten Lebensstiltyps gegenüber den anderen Jugendlichen.

Tabelle 3: Clusterzentren der Variablen der Vier-Cluster-Lösung

\begin{tabular}{|c|c|c|c|c|}
\hline & Cl 1 & Cl 2 & Cl 3 & Cl 4 \\
\hline & $n=223$ & $n=175$ & $n=147$ & $n=63$ \\
\hline \multicolumn{5}{|l|}{ Lebensstilelemente (z-Werte) } \\
\hline Freizeitverhalten: Ausgehen & -.08 & .27 & -.46 & .60 \\
\hline Kulturelle Aktivitäten & .42 & -.15 & -.28 & -.44 \\
\hline Kreative Aktivitäten & .61 & -.49 & -.22 & -.25 \\
\hline Computer- und Internetnutzung & -.51 & .38 & .01 & .73 \\
\hline Film- und Fernsehkonsum: Actionformat & -.66 & .48 & .10 & .75 \\
\hline Informationsformat & .21 & -.29 & -.14 & .20 \\
\hline Unterhaltungsformat & .26 & .23 & -.66 & -.03 \\
\hline Musikkonsum: Moderne Tanzmusik & -.23 & .53 & -.35 & .19 \\
\hline Provokative Jugendmusik & -.02 & -.23 & .31 & -.01 \\
\hline Anspruchsvolle Musik & .45 & -.35 & -.22 & -.09 \\
\hline
\end{tabular}




\begin{tabular}{|l|r|r|r|r|}
\hline Kleidungsstil: Hip/flippig & .21 & .33 & -.61 & -.23 \\
\hline Qualitätsbewusst/markenorientiert & -.09 & .53 & -.70 & .49 \\
\hline Salopp & .46 & -.14 & -.31 & -.51 \\
\hline Zeitschriftenkonsum: Zeitgeschehen & .38 & -.30 & -.23 & .03 \\
\hline Männerthematik & -.38 & -.17 & -.18 & 2.27 \\
\hline Fachzeitschriften & -.68 & .50 & .05 & .90 \\
\hline Jugendzeitschriften & .02 & .26 & -.30 & -.09 \\
\hline Unterhaltung & .01 & .36 & -.40 & -.12 \\
\hline Einrichtungsstil: Ordentlich & .08 & .56 & -.73 & -.16 \\
\hline Kreativ & .27 & .44 & -.74 & -.47 \\
\hline Bequem & .01 & .46 & -.48 & -.19 \\
\hline Soziodemographie (Angaben in \%) & & & & \\
\hline Geschlecht: Weiblich & 88.8 & 34.9 & 18.4 & 6.3 \\
\hline Männlich & 11.2 & 65.1 & 81.6 & 93.7 \\
\hline Besuchte Schulform: Hauptschule & 12.1 & 48.0 & 27.7 & 58.7 \\
\hline Realschule & 32.7 & 29.7 & 31.3 & 19.0 \\
\hline Gymnasium & 50.7 & 18.9 & 35.4 & 20.6 \\
\hline Bildungsabschluss Mutter: keiner & 1.8 & 1.2 & 0.7 & 6.5 \\
\hline Hauptschulabschluss & 26.6 & 32.7 & 28.8 & 43.5 \\
\hline Realschulabschluss & 46.8 & 49.1 & 45.2 & 29.0 \\
\hline Abitur & 7.7 & 4.1 & 8.9 & 9.7 \\
\hline Studium & 14.9 & 10.5 & 10.3 & 9.7 \\
\hline Bildungsabschluss Vater: keiner & 4.1 & 4.8 & 2.8 & 8.1 \\
\hline Hauptschulabschluss & 26.2 & 41.1 & 38.2 & 37.1 \\
\hline Realschulabschluss & 36.2 & 27.4 & 27.8 & 29.0 \\
\hline Abitur & 12.2 & 12.5 & 11.1 & 12.9 \\
\hline Studium & 19.0 & 9.5 & 12.5 & 9.7 \\
\hline
\end{tabular}

Die vier Lebensstile lassen sich wie folgt bezeichnen:

Cl 1: Hochkultureller Lebensstil. Dieser mädchen- und gymnasiastendominante Lebensstil ist insbesondere durch eine stark überdurchschnittliche Beschäftigung mit Kulturgütern geprägt. Kulturelle und kreative Tätigkeiten stellen Hauptfreizeitbeschäftigungen dar. Die actionformatbezogene audiovisuelle Mediennutzung wird abgelehnt. Der bevorzugte Musikstil ist anspruchsvolle Musik. Fachzeitschriften stellen ein deutliches Distinktionsmittel dar.

Cl 2: Darstellender Lebensstil. Diese noch am ehesten geschlechtsparitätische, haupt- und realschulstarke Lebensstilgruppe zeichnet sich insbesondere durch die Einrichtungsstile sowie einer Präferenz für moderne Tanzmusik und eines markenorientierten Kleidungsstils aus. Die höchste Distinktion besteht gegenüber kreativen Freizeitbeschäftigungen.

Cl 3: Zurückhaltender Lebensstil. Die jungendominante, bildungsdurchschnittliche Lebensstilgruppe ist insgesamt am ehesten durch Zurückhaltung bzw. Unauffälligkeit gekenn- 
zeichnet. Auffällig für diese Gruppe stellen sich die vielen distinktiven Stilelemente dar. Es gibt keine einzige überdurchschnittlich positive Stilisierungsausprägung. Überdurchschnittlich distinkt ausgeprägt sind hingegen das Ausgehen in der Freizeit, der unterhaltungsformatbezogene Film- und Fernsehkonsum, ein hipper sowie markenorientierter Kleidungsstil sowie ein ordentlicher, kreativer und bequemer Einrichtungsstil.

Cl 4: Hedonistischer Lebensstil. Der jungen- und hauptschuldominante Lebensstil ist vor allem durch ein erlebnis- und lustbezogenes Verhalten charakterisiert. Besonders stark sind in dieser Lebensstilgruppe das Ausgehen in der Freizeit, die Computer- und Internetnutzung, die Medienpräferenz des audio-visuellen Actionformats, das Lesen von männerthematischen Zeitschriften sowie Fachzeitschriften ausgeprägt. All diese Merkmale stellen sich im hedonistischen Lebensstil im Vergleich zu den anderen Lebensstilen als am stärksten ausgebildet dar. Eine deutliche Abgrenzung findet hingegen zu kulturellen Aktivitäten in der Freizeit, einem saloppen Kleidungsstil und einem kreativen Einrichtungsstil statt.

\subsection{Lebensstile und gesundheitsrelevante Verhaltensweisen}

Um die Bedeutung der einzelnen gesundheitsrelevanten Verhaltensweisen in den unterschiedlichen Lebensstilgruppen zu ermitteln, wurden Kreuztabellierungen berechnet (s. Tab. 4).

Tabelle 4: Kreuztabellierung für Lebensstile und gesundheitsrelevante Verhaltensweisen (Angaben in \% für ,oft" bis ,,sehr oft")

\begin{tabular}{|l|l|l|l|l|l|}
\hline & $\begin{array}{c}\text { Exponiertes } \\
\text { Risikover- } \\
\text { halten }\end{array}$ & $\begin{array}{c}\text { Proaktives } \\
\text { Gesund- } \\
\text { heitsver- } \\
\text { halten }\end{array}$ & $\begin{array}{c}\text { Gesundheits- } \\
\text { riskantes Sozi- } \\
\text { alkontaktver- } \\
\text { halten }\end{array}$ & $\begin{array}{c}\text { Explizit risi- } \\
\text { ko-konnota- } \\
\text { tive } \\
\text { Aktivitäten }\end{array}$ & $\begin{array}{c}\text { Verdecktes } \\
\text { gesundheit- } \\
\text { liches Risi- } \\
\text { koverhalten }\end{array}$ \\
\hline Hochkultureller LS & 0.4 & 63.2 & 5.8 & 2.7 & 12.6 \\
\hline Darstellender LS & 0.6 & 42.9 & 26.8 & 11.4 & 17.8 \\
\hline Zurückhaltender LS & 2.8 & 39.5 & 12.2 & 3.4 & 6.2 \\
\hline Hedonistischer LS & 8.0 & 38.1 & 31.8 & 14.2 & 14.2 \\
\hline Chi $^{2}(d f)$ & $47.2(12)^{* *}$ & $58.9(12)^{* * *}$ & $113.7(12)^{* *}$ & $64.5(12)^{* *}$ & $21.9(12)^{+}$ \\
\hline Eta & .24 & .26 & .37 & .27 & .09 \\
\hline
\end{tabular}

$\mathrm{p}={ }^{+}<.05 * *<.001$

Der hochkulturelle Lebensstil erweist sich als der gesundheitlichförderlichste Lebensstil. Knapp zwei Drittel der Jugendlichen innerhalb der hochkulturellen Lebensstilgruppe gaben an, sich überwiegend gesundheitlich proaktiv zu verhalten. Hingegen praktizieren ,hochkulturelle" Jugendliche bis auf das verdeckte gesundheitliche Risikoverhalten nur im unteren einstelligen Prozentbereich gesundheitsriskante Verhaltensrepertoires. Die nächste sich insgesamt am gesundheitlichförderlichsten verhaltende Gruppe ist die zurückhaltende Lebensstilgruppe. In der Präsenz des proaktiven Verhaltens bestehen nur kleine Differenzen zur darstellenden und hedonistischen Lebensstilgruppe, aber in der Ausübung gesundheitsriskanter Praktiken besteht insgesamt ein deutlicher Unterschied zugunsten der ,zurückhaltenden“ Jugendlichen. Als gesundheitlich stärker gefährdend stellt sich der darstellende Lebensstil und in einer noch höheren Ausprägung der hedonistische Lebensstil dar. Unter den „hedonistischen“ Jugendlichen gaben knapp ein Drittel an, dass sie oft bzw. sehr oft laute Musik hören, Cannabis- und Tabakprodukte sowie Alkohol konsumieren (gesundheitsris- 
kantes Sozialkontaktverhalten). Zudem gab jeder siebte „hedonistische“ Jugendliche eine hohe Affinität zu explizit risiko-konnotativen Aktivitäten (ERKA) an und immerhin $8 \%$ berichteten über exponiertes Risikoverhalten.

\subsection{Die gesundheitsrelevante Bedeutung einzelner Lebensstilbereiche}

In diesem Abschnitt werden die unterschiedlichen Lebensstilbereiche/-elemente in ihrer Bedeutung für die eben dargestellten gesundheitsrelevanten Verhaltensdimensionen ermittelt. Dies erfolgt anhand multipler schrittweiser Regressionsanalysen (s. Tab. 5). Diese Methode nimmt nicht alle Regressoren (Prädikatoren) in das Modell auf, sondern nur diejenigen, die sich als relevant erweisen, indem sie ein bestimmtes Gütekriterium maximieren. Somit ist das Regressionsmodell ausschließlich aus statistisch signifikanten Regressoren bestimmt. Allerdings wurden die gesundheitsrelevanten Verhaltensdimensionen ,exponiertes Risikoverhalten“ (Fak. 1) und „verdecktes gesundheitliches Risikoverhalten“ (Fak. 5) nicht mehr weiter in der Analyse berücksichtigt, da für diese nur sehr geringe Aufklärungsquoten ermittelt werden konnten.

Tabelle 5: Gesundheitsrelevante Verhaltensdimensionen und Lebensstilbereiche

\begin{tabular}{|c|c|c|c|c|c|c|}
\hline & \multicolumn{2}{|c|}{$\begin{array}{l}\text { Proaktives Gesund- } \\
\text { heitsverhalten }\end{array}$} & \multicolumn{2}{|c|}{$\begin{array}{l}\text { Gesundheitsriskantes } \\
\text { Sozialkontaktverhalten }\end{array}$} & \multicolumn{2}{|c|}{$\begin{array}{l}\text { Explizit risiko-konno- } \\
\text { tative Aktivitäten }\end{array}$} \\
\hline & $\mathrm{B}$ & beta & B & beta & B & beta \\
\hline Freizeit: Ausgehen & - & - & .30 & $.29 * *$ & .12 & $.13 * *$ \\
\hline kulturelle Aktivitäten & .11 & $.11^{*}$ & - & - & - & - \\
\hline kreative Aktivitäten & - & - & -.13 & $-.10 * *$ & - & - \\
\hline Computer/Internet & -.11 & $-.16^{* *}$ & .06 & $.08 *$ & - & - \\
\hline $\begin{array}{l}\text { Film-/Fernsehkonsum: } \\
\text { Actionformat }\end{array}$ & -.17 & $-.18 * *$ & .24 & $.22 * *$ & .27 & $.26^{* *}$ \\
\hline Informationsformat & - & - & -.18 & $-.15 * *$ & - & - \\
\hline $\begin{array}{l}\text { Musik: } \\
\text { moderne Tanzmusik }\end{array}$ & -.06 & $-.09 *$ & - & - & - & - \\
\hline anspruchsvolle Musik & - & - & -.17 & $-.13 * *$ & - & \\
\hline Kleidung: hip/flippig & - & - & .23 & $.20 * *$ & - & \\
\hline markenorientiert & .11 & $.11^{*}$ & - & - & - & \\
\hline $\begin{array}{l}\text { Zeitschriften: Zeitge- } \\
\text { schehen }\end{array}$ & .11 & $.12 * *$ & - & - & - & - \\
\hline "Männerthematik" & -.11 & $-.10 *$ & .22 & $.16^{* *}$ & .13 & $.10^{*}$ \\
\hline Fachzeitschriften & .08 & $.10^{*}$ & - & - & - & - \\
\hline Jugendzeitschriften & - & - & -.14 & $-.14 * *$ & - & - \\
\hline Unterhaltung & - & - & - & - & .11 & $.12 * *$ \\
\hline $\begin{array}{l}\text { Einrichtungsstil: ordent- } \\
\text { lich/sachlich }\end{array}$ & .14 & $.16^{* *}$ & - & - & -.14 & $-.14 * *$ \\
\hline$R 2$ & \multicolumn{2}{|c|}{$.22 * *$} & \multicolumn{2}{|c|}{$.43 * *$} & \multicolumn{2}{|c|}{$.17 * *$} \\
\hline
\end{tabular}

$\mathrm{p}=*<.01 ; * *<.001$ 
Mit dem proaktiven Gesundheitsverhalten stehen 9 Lebensstilbereiche in einem statistisch signifikanten Zusammenhang. An der Spitze finden sich der Konsum des audiovisuellen Actionformats, die Computer-/Internetnutzung sowie ein ordentlicher Einrichtungsstil. Hierbei stehen die beiden erstgenannten Prädiktoren in einem negativen Zusammenhang und erweisen sich so als gesundheitsabträglich. Eine sachliche Gestaltung der eigenen vier Wände geht mit dem proaktiven Gesundheitsverhalten einher. Positiv korrelieren weiterhin das Interesse für politisches Zeitgeschehen, ein marken- bzw. qualitätsorientierter Kleidungsstil, kulturelle Freizeitaktivitäten und eine Vorliebe für Fachzeitschriften. In einem schwach negativen Zusammenhang stehen „männerthematische“ Zeitschriften und die Präferenz von moderner Tanzmusik. Diese Stilisierungen sind somit als gesundheitsabträglich zu bezeichnen. Bei den negativ korrespondierenden „männerthematischen“ Zeitschriften, actionbezogenen Filmund Fernsehkonsum sowie der Computer- und Internetnutzung ist von einer Konfundierung mit dem Faktor Geschlecht auszugehen. Die anderen Stilisierungen sind allerdings relativ geschlechtsneutral ausgeprägt. Mit den herauskristallisierten Lebensstilelementen können $22 \%$ der Varianz des proaktiven Gesundheitsverhaltens aufgeklärt werden.

Für das gesundheitsriskante Sozialkontaktverhalten findet sich der „höchste“ Zusammenhangswert für das Ausgehen in der Freizeit. Dieser Befund überrascht letztendlich nicht, denn es handelt sich bei diesen gesundheitsriskanten Verhaltensweisen um solche, die sozialkontaktförderlich sind. Eine Kontaktaufnahme mit den gleich- oder auch andersgeschlechtlichen Altersgenossen ist vor allem ,Sinn und Zweck“ des Ausgehens. Hierbei wird die Gefährlichkeit dieser gesundheitsrelevanten Verhaltensweisen deutlich. In einem positiven Zusammenhang stehen weiterhin der audiovisuelle Actionkonsum, ein flippiger Kleidungsstil und die „männerthematische“ Zeitschriftenpräferenz. Eine negative Korrelation besteht hingegen mit dem Informationsformat in Film und Fernsehen, der Vorliebe für Jugendzeitschriften, einem anspruchsvollen Musikgeschmack und mit einer kreativen Freizeitbeschäftigung. All diese Stilelemente erweisen sich als gesundheitspräventive Größen. Ein schwacher positiver Zusammenhang kann noch für die Computer-/Internetnutzung festgestellt werden. Mit den 9 Lebensstilelementen lassen sich $43 \%$ (!) des gesundheitsriskanten Sozialkontaktverhaltens aufklären.

Die explizit risiko-konnotativen Verhaltensweisen lassen sich zu 17\% aufklären. Hauptkorrelat ist hier das Actionformat. In einem positiven Zusammenhang stehen weiterhin das Ausgehen sowie das Lesen von Unterhaltungszeitschriften und ,,männerthematischen“ Zeitschriften. Als gesundheitsrelevante Ressource ist ausschließlich ein ordentlich/sachlicher Einrichtungsstil zu ermitteln. Von einer geschlechtsspezifischen Konfundierung kann bei dem Actionkonsum und den „,männerthematischen“ Zeitschriften ausgegangen werden. Die anderen Stilisierungen sind hingegen geschlechtsneutral.

\section{Zusammenfassung und Diskussion}

Die Befunde zeigen sehr deutlich lebensstilistische Differenzen bezüglich des gesundheitsrelevanten Verhaltens. Bedeutende Beziehungen ließen sich zwischen einzelnen Lebensstilelementen und den gesundheitsrelevanten Verhaltensweisen aufdecken. Die höchste Varianzaufklärung ließ sich für das gesundheitsriskante Sozialkontaktverhalten feststellen, womit hier auf eine hohe lebensstilistische Verankerung geschlossen werden kann. Das kann gleichfalls als Beleg für die lebensstilistische und gleichaltrigengruppenbezogene Verknüpfung des Tabak- und Alkoholkonsums im Jugendalter gesehen werden. Dies ist umso bedeutender, da insbesondere diese Verhaltensweisen (Alkohol-/Tabakkonsum) die höchsten Prävalenzraten innerhalb der gesundheitsriskanten Verhaltensformen im Jugendalter haben (vgl. BZgA 2001). 
Qualitative Untersuchungen zeigen, dass der Hauptgrund für Jugendliche zu trinken in der erleichterten sozialen Integration in die Gruppe der Gleichaltrigen liegt (vgl. Pavis/Cunningham-Burley/Amos 1997). Gemessen an der Häufigkeit und der erlebten Intimität von Kontakten in Beziehungsgefügen lässt sich für konsumierende Jugendliche in der Tendenz eine stärkere soziale Einbindung feststellen (vgl. Kandel/Davies 1991). Bei Mädchen zeigt sich, dass diejenigen, die rauchen, über einer größere Anzahl an Freunden und Freundinnen verfügen als nichtrauchende Mädchen (vgl. Collins 1993). Soziale Unterstützung durch Gleichaltrige ist folglich weniger ein Schutzfaktor als ein Risikofaktor für den Substanzkonsum (vgl. Schmidt 1998). „Überdurchschnittlicher Substanzkonsum (Alkohol und Zigaretten) steht im Zusammenhang mit Lebensstilen, die meist von überdurchschnittlichen Sozialkontakten zu Gleichaltrigen, geselliger Freizeitbeschäftigung, konkreter Jugendorientierung und sozialen Fähigkeiten gekennzeichnet ist" (Schmidt 1998, 55). Als besonders relevant für den Substanzkonsum haben sich der hedonistische und in protektiver Richtung der hochkulturelle Lebensstil herausgestellt.

Im Folgenden zu den einzelnen Lebensstilen: Der hochkulturelle Lebensstil hat sich als vornehmlich gesundheitsförderlich, präventiv und protektiv herausgestellt. Dies mag vor allem auch mit der Konstituierung dieser Lebensstilgruppe zusammenhängen, da in ihr der größte Mädchen- und Gymnasiastenanteil vertreten ist. In diesem Kontext sind auch die gesundheitszuträglichen Stilisierungen kultureller und kreativer Freizeitaktivitäten sowie anspruchsvoller Musik und Zeitgeschehen (Printmedien) eingebunden. In sozialstruktureller Hinsicht konnte eine hoch bildungspartizipative herkunftsfamiliäre Soziallage konstatiert werden. Die Eltern der hochkulturell lebenden Jugendlichen verfügen über die höchsten Bildungsabschlüsse und unter ihnen sind die meisten Akademiker zu finden. Diese sozialstrukturelle Ausgangslage erweist sich für die Folgegeneration als hochgradig pro-gesundheitlich.

Für den darstellenden Lebensstil, in dem der zweithöchste Mädchen- sowie auch Hauptschüleranteil vertreten ist, konnten stärkere gesundheitsabträgliche Zusammenhänge festgestellt werden. Zwischen dem gesundheitsriskanten Sozialkontaktverhalten und einem auf Darstellung akzentuierten Stilisierungsverhalten lassen sich Wechselbeziehungen bestätigen. Innerhalb der gesundheitsrelevanten Lebensstilbereiche sind einerseits die gesundheitsabträglichen Elemente moderner Tanzmusik, flippiger Kleidungsstil und das Lesen von Unterhaltungsmagazinen und andererseits die gesundheitsförderlichen Stilisierungselemente ordentlicher und bequemer Einrichtung für den darstellenden Lebensstil charakteristisch. Sozialstrukturell haben die Väter dieser Jugendlichen zum größten Teil einen Hauptschulabschluss und die Mütter einen Realschulabschluss. Aus dieser eher traditional-materialistischen Soziallage heraus ist auch das gesundheitsriskante Sozialkontaktverhalten zu verstehen. Denn so gilt es doch als (tradierte) Konvention, zu sozialen Anlässen beispielsweise auch zu trinken. Dieser Logik folgend, kann das gesundheitsriskante Sozialkontaktverhalten als ein Ausdruck konservativ-traditionaler Haltung verstanden werden.

Der zurückhaltende Lebensstil ist wiederum stärker mit gesundheitsförderlichen Verhaltensweisen assoziiert. Soziodemographisch lässt sich für diese Lebensstilgruppe der zweitgrößte Jungenanteil (82\%) und eine gleichmäßige Bildungsverteilung entsprechend der Stichprobe feststellen. Lebensstilistisch fällt auf, dass die als gesundheitsrelevant ermittelten Lebensstilelemente in diesem Lebensstil alle als Distinktionsmittel fungieren und keine positive Ausprägung besitzen. Ein protektiver Zusammenhang ergibt sich aus der Ablehnung des Ausgehens, der modernen Tanzmusik, des flippigen Kleidungsstils und der Unterhaltungsmagazine. Das Bildungsniveau der Eltern entspricht ohne große Abweichungen und Auffälligkeiten dem Durchschnitt der Stichprobe.

Der hedonistische Lebensstil stellt den Gegenpol zum hochkulturellen Lebensstil dar und ist als am gesundheitsriskantesten zu bezeichnen. Da eine riskante Verhaltensausrichtung vor al- 
lem unter männlichen Jugendlichen zu beobachten und dies auch noch bildungsspezifisch umformt ist (vgl. Helfferich 1997), verwundert es nicht, dass die hedonistische Lebensstilgruppe zu 94\% aus Jungen besteht und ihr der größte Hauptschüleranteil angehört. Die Lebensstilgruppe stellt sich somit als eine recht homogene Jugendlichengruppe dar. Innerhalb der gesundheitsrelevanten Lebensstilelemente treten vor allem das Ausgehen, die Computer- und Internetnutzung, der actionbezogene Film- und Fernsehkonsum und die „männerthematischen“ Zeitschriften als Charakteristik des hedonistischen Lebensstils heraus. In sozialstruktureller Hinsicht ist in der Herkunftsfamilie eine unterdurchschnittliche Bildungspartizipation festzustellen, zudem sind hier die meisten Eltern ohne Schulabschluss anzufinden.

Die Studie hatte die Absicht, anhand von Lebensstilen resp. alltagsästhetischen Stilisierungen bedeutsame Merkmale gesundheitsrelevanter Verhaltensweisen zu ermitteln. Es konnten in der Untersuchung zwei prototypische gesundheitsbezogene Lebensstile identifiziert werden. Auf der einen Seite der gesundheitsförderliche und protektive hochkulturelle Lebensstil und auf der anderen Seite der gesundheitsriskante hedonistische Lebensstil. Die diese Lebensstile substanziell konstituierenden Stilisierungen erwiesen sich hierbei als generelle gesundheitsförderliche oder gesundheitsriskante Lebensstilbereiche (vgl. Tab. 3). Bemerkenswert ist darüber hinaus, dass sich auch der darstellende und zurückhaltende Lebensstil relativ eindeutig einem eher gesundheitsförderlichen oder gesundheitsriskanten Verhaltensspektrum zuordnen lässt.

Anhand der Lebensstiltypologie resp. der alltagsästhetischen Stilisierungselemente konnte die höchste Varianzaufklärung für das gesundheitsriskante Sozialkontaktverhalten erzielt werden. Ebenfalls sind die Varianzaufklärungen für das proaktive Gesundheitsverhalten und die explizit risiko-konnotativen Aktivitäten bemerkenswert. Hierin spiegelt sich die lebensstilistische Eingebundenheit der gesundheitsrelevanten Verhaltensweisen wider. Dieser Erkenntnisgewinn ist insbesondere in präventiver Hinsicht überaus wichtig und perspektiveröffnend (bereits Jessor und Jessor (1977) forderten, den Lebensstil der Jugendlichen für zukünftige Präventions-/Interventionsbemühungen stärker zu berücksichtigen). Hingegen waren für das verdeckte gesundheitliche Risikoverhalten und exponierte Risikoverhalten keine nennenswerten lebensstilistischen Verbindungen festzustellen.

Mit den gesundheitsförderlichen vs. gesundheitsriskanten Stilisierungsmerkmalen gehen prototypische Geschlechtsrollenorientierungen einher. Dies erweist sich idealtypisch anhand der Polarisation von hochkulturellem vs. hedonistischem Lebensstil. So sind auf der einen Seite mit dem mädchendominanten hochkulturellen Lebensstil und entsprechenden Stilisierungen gesundheitsförderliche Verhaltensweisen verbunden, während auf der anderen Seite mit dem jungendominanten hedonistischen Lebensstil und seinen markanten Stilisierungen gesundheitsriskante Verhaltensweisen verknüpft sind. Somit zeigen sich auch in der Lebensstilanalyse geschlechtsspezifische Dichotomien, die sich im gesundheitsrelevanten Verhalten fortsetzen (vgl. Helfferich 1997; Kolip 1997a).

Doch ist bei dieser Dichotomie nicht zu übersehen, dass sich in den beiden anderen Lebensstilgruppen keine so eindeutigen und homogenen Gruppen identifizieren lassen. Hier gibt es eher Jungen, die sich nicht entsprechend eines prototypischen Bildes von Männlichkeit verhalten (der „Marlboro-Mann“ - vgl. Sieverding 1997), wie auch Mädchen, die sich nicht stereotyp weiblich verhalten. Es manifestiert sich die Existenz von Geschlechtermodellen. Das heißt, dass sich hier vor allem die femininen Jungen und die maskulinen Mädchen finden (vgl. Sieverding 2000; Raithel 2003a). Diese intrageschlechtlichen Differenzen sind in präventiver Hinsicht im Hinterkopf zu behalten. Der präventive und gesundheitsfördernde Hauptansatz sollte jedoch erst einmal die geschlechtsrollenprototypischen Lebensstilisierungen und Eingebundenheit gesundheitsförderlichen vs. gesundheitsriskanten 
Verhaltens in den Mittelpunkt rücken. Aus dieser Perspektive wird Gesundheitserziehung zu einem guten Stück Geschlechterarbeit.

Neben der Geschlechtsperspektive sollte auch die soziale Ungleichheitsperspektive berücksichtigt werden. Die Befunde bestätigen eindeutige Unterschiede im gesundheitsrelevanten Verhalten im Zusammenhang mit dem herkunftsfamiliären Bildungsstatus, welcher als harter Ungleichheitsindikator gilt. Hierbei verhalten sich die Jugendlichen höher gebildeter Eltern gesundheitsförderlicher als ihre Altersgenossen, deren Eltern einen niedrigeren oder überhaupt keinen Schulabschluss besitzen. Für diese gesundheitliche Ungleichheit sind jedoch nicht nur sozioökonomische Unterschiede verantwortlich zu machen, sondern es gehen mit der Bildung auch mentalitätsspezifische Unterschiede einher, die in der Herkunftsfamilie nachhaltig prägend wirken. Somit gilt es in der Ungleichheitsperspektive immer die ökonomische und mentale Dimension zu berücksichtigen.

In der Geschlechts- und Ungleichheitsperspektive sind in Bezug auf die Ausübung gesundheitsriskanter Verhaltensweisen insbesondere die männlichen Hauptschüler (hedonistische Lebensstilgruppe) aufgefallen. Hiermit lassen sich geschlechts- und ,schichtspezifische" Unterschiede entsprechend der These Helfferichs (1997) der kulturellen Symbolik des Bereichs der Extremkategorien bestätigen (vgl. auch Liebau 1992; Moore 1993; Vester/Gardemin 2000; Dumais 2002).

Durch die Lebensstilanalyse ist das Spektrum gesundheitsrelevanter Verhaltensweisen zu einem großen Teil als ein „Verhaltens-Syndrom“ (vgl. Jessor/Jessor 1977) mit Lebensweltbezügen im Sinne Franzkowiaks (1986) zu bestätigen. Die Befunde führen zu dem Schluss, dass gesundheitsrelevante Verhaltensweisen in den Lebensstil eingebunden sind, worüber Geschlechtszugehörigkeit reproduziert werden kann (vgl. Raithel 2004). Dieser Schluss ist auch gegenüber der Beobachtung, dass sich die Geschlechter im Alkohol- und Tabakkonsum quantitativ immer mehr angleichen, haltbar, da sich Mädchen und Jungen in der Qualität des Konsums deutlich unterscheiden (vgl. Kolip 1997b; Sieverding 2000).

Somit wäre es durchaus sinnvoll, ,gesundheitsbezogene“ Lebensstile zu definieren (vgl. Abel 1999). Dies gilt insbesondere für das Jugendalter, da gesundheitliches Risikoverhalten in dieser Lebensphase während des Erprobens und Austestens von Grenzen eine überaus große Rolle spielt (vgl. Raithel 2004). Allerdings dürften allgemeine lebensstilistische Merkmale den gesundheitsrelevanten Verhaltensweisen nicht nachstehen. Aus erhebungsökonomischen Gründen sollte jedoch eine zu hohe Anzahl von Lebensstilindikatoren vermieden werden. Bei der Auswahl einzubeziehender gesundheitsrelevanter Lebensstilmerkmale wäre auf eine gewisse Kontinuität der Verhaltensweisen (situationsübergreifende Verhaltenstendenzen) zu achten.

Abschließend sei noch in methodischer Hinsicht zu den Selbstauskunftsdaten angemerkt, dass natürlich die Frage nach der Authentizität und „Wahrheit“ oder „Objektivität“ offen bleibt, da dies ein methodisches Grundproblem sozialwissenschaftlicher Erkenntnisgewinnung und insbesondere von Befragungen darstellt. Dies bezieht sich auch auf die Frage, inwiefern geschlechtsspezifisches Antwortverhalten eine Rolle spielte und zu Verzerrungen führte, indem möglicherweise die Mädchen bei Risikoverhaltensweisen eher untertrieben und die Jungen eher übertrieben haben (vgl. Sieverding 2000). Somit ist von einer gewissen Über- bzw. Untertreibung auszugehen, welche allerdings die subjektive Konstruktion der Wirklichkeit widerspiegelt (vgl. Kolip 1997a).

Bezüglich der Güte der Lebensstiltypologie zeigt sich, dass für die ermittelten Lebensstile eine überraschend große Deckung mit bereits bestehenden Lebensstiltypologien im Jugendalter besteht (vgl. Ulbrich-Herrmann 1998; Georg 1992; 1998). Somit können die hier ermit- 
telten Lebensstile einerseits die Ergebnisse anderer Lebensstiluntersuchungen bestätigen und andererseits ihre eigene empirische Relevanz unter Beweis stellen.

\section{Literatur}

Abel, T. (1991): Measuring Health Life-styles in a Comparative Analysis: Theoretical Issues and Emirical Findings. In: Soc.Sci.Med., 32, 899-908.

Abel, T. (1999): Gesundheitsrelevante Lebensstile. Zur Verbindung von handlungs- und strukturtheoretischen Aspekten in der modernen Ungleichheitsforschung. In: Maeder, C./Burton-Jeangros, C./HaourKnipe, M. (Hrsg.): Gesundheit, Medizin und Gesellschaft. Beiträge zur Soziologie der Gesundheit. Zürich: Seismo, 43-61.

Abel, T./Rütten, A. (1994): Struktur und Dynamik moderner Lebensstile: Grundlagen für ein neues empirisches Konzept. In: Dangschat, J.S./Blasisus, J. (Hrsg.): Lebensstile in den Städten. Opladen: Leske + Budrich, 216-234.

Allmer, H. (1990): Gesundheitsverhalten als intentionales und volitives Geschehen. In: Schwarzer, R. (Hrsg.): Gesundheitspsychologie. Ein Lehrbuch. Göttingen: Hogrefe, 117-130.

Angermeyer, M.C./Klusmann, D. (1987): From Social Class to Social Stress. In: Angermeyer, M.C. (Ed.): From Social Class to Social Stress. New Developments in Psychiatric Epidemiology. Berlin: Springer, 2-13.

Bacher, J. (1996): Clusteranalyse. Anwendungsorientierte Einführung. München: Oldenbourg Verlag.

Backhaus, K./Erichson, B./Plinke, W./Weiber, R. (1990): Multivariate Analysemethoden. Eine anwendungsorientierte Einführung. Berlin: Springer.

Becker, U./Nowak, H. (1982): Lebensweltanalyse als neue Perspektive der Markt- und Meinungsforschung. In: E.S.O.M.A.R. Kongreß, Band 2, 247-267.

Bourdieu, P. (1982): Die feinen Unterschiede. Zur Kritik der gesellschaftlichen Urteilskraft. Frankfurt: Suhrkamp.

BZgA - Bundeszentrale für gesundheitliche Aufklärung (2001): Die Drogenaffinität Jugendlicher in der Bundesrepublik Deutschland 2001. Eine Wiederholungsbefragung. Köln: Bundeszentrale für gesundheitliche Aufklärung.

Collins, R.E. (1993): Women's Issues in Alcohol Use and Cigarette Smoking. In: Baer, J.S./Marlatt, G.A./ McMahon, J. (Eds.): Addictive Behaviors Across the Life Span. Newbury Park: Sage, 274-307.

Dumais, S.A. (2002): Cultural Capital, Gender, and School Success: The Role of Habitus. In: Sociology of Education, 75, 44-68.

Elkeles, T./Mielck, A. (1997): Entwicklung eines Modells zur Erklärung gesundheitlicher Ungleichheit. In: Gesundheitswesen, 59, 137-143.

Fischer, A. (1992) (Red.): Jugend '92. Lebenslagen, Orientierungen und Entwicklungsperspektiven im vereinigten Deutschland. Band 4. Opladen: Leske + Budrich.

Franzkowiak, P. (1986): Risikoverhalten und Gesundheitsbewußtsein bei Jugendlichen. Der Stellenwert von Rauchen und Alkoholkonsum im Alltag von 15 bis 20jährigen. Berlin: Springer.

Gerhards, J./Rössel, J. (2002): Lebensstile und ihr Einfluss auf das Ernährungsverhalten von Jugendlichen. In: Soziale Welt, 53, 3, 261-284.

Georg, W. (1992): Jugendliche Lebensstile - ein Vergleich. In: Zinnecker, J. (Hrsg.): Jugend '92. Lebenslagen, Orientierungen und Entwicklungsperspektiven im vereinten Deutschland. Band 2 - Im Spiegel der Wissenschaften. Opladen: Leske + Budrich, 265-286.

Georg, W. (1998): Soziale Lage und Lebensstil. Eine Typologie. Opladen: Leske + Budrich.

Gluchowski, P. (1987): Feizeit und Lebensstil. Plädoyer für eine integrierte Analyse von Freizeitverhalten. Erkrath: DGFF.

Hartmann, P.H. (1999): Lebensstilforschung. Darstellung, Kritik und Weiterentwicklung. Opladen: Leske + Budrich. 
Helfferich, C. (1997): „Männlicher“ Rauschgewinn und „,weiblicher“ Krankheitsgewinn? Geschlechtsgebundene Funktionalität von Problemverhalten und die Entwicklung geschlechtsbezogener Präventionsansätze. In: Zeitschrift für Sozialisationsforschung und Erziehungssoziologie, 17, 2, 148-161.

Hradil, S. (1987): Sozialstrukturanalyse einer fortgeschrittenen Gesellschaft. Opladen: Leske + Budrich.

Hradil, S. (1992): Alte Begriffe und neue Strukturen. Die Milieu-, Subkultur- und Lebensstilforschung der 80er Jahre. In: Hradil, S. (Hrsg.): Zwischen Bewußtsein und Sein. Die Vermittlung „objektiver“ Lebensbedingungen und ,subjektiver“ Lebensweisen. Opladen: Leske + Budrich, 15-55.

Hradil, S. (1999): Soziale Ungleichheit in Deutschland. Opladen: Leske + Budrich.

Hradil, S. (2001): Eine Alternative? Einige Anmerkungen zu Thomas Meyers Aufsatz „Das Konzept der Lebensstile in der Sozialstrukturforschung“. In: Soziale Welt, 52, 273-282.

Hurrelmann, K. (1994): Lebensphase Jugend. Eine Einführung in die sozialwissenschaftliche Jugendforschung. Weinheim: Juventa.

Hurrelmann, K. (2000): Gesundheitssoziologie. Eine Einführung in sozialwissenschaftliche Theorien von Krankheitsprävention und Gesundheitsförderung. Weinheim: Juventa.

Jessor, R./Jessor, L. (1977): Problem Behavior and Psychological Development: A Longitudinal Study of Youth. New York: Adademic Press.

Kandel, D./Davies, M. (1991): Friendship, Network, Intimacy, and Illicit Drug Use in Young Adulthood. In: Criminology, 29, 3, 441-469.

Kasl, S.V./Cobb, S. (1966): Health Behavior, Illnes, and Sick-role Behavior. In: Archives of Environmental Psychology, 12, 246-266, 531-541.

Klein, T./Schneider, S./Löwel, H. (2001): Bildung und Mortalität. Die Bedeutung gesundheitsrelevanter Aspekte des Lebensstils. In: Zeitschrift für Soziologie, 30, 384-400.

Kolip, P. (1995): Ernährung und Körperzufriedenheit: Der Einfluß von Alter und Geschlecht auf Körperzufriedenheit und Ernährungsverhalten im Jugendalter. In: Zeitschrift für Gesundheitspsychologie, III, 2, 97-113.

Kolip, P. (1997a): Geschlecht und Gesundheit im Jugendalter. Die Konstruktion von Geschlechtlichkeit über somatische Kulturen. Opladen: Leske + Budrich.

Kolip, P. (1997b): Das gleiche ist nicht dasselbe: Zur Notwendigkeit geschlechtsspezifischer Suchtprävention im Jugendalter. In. Unterrichtswissenschaft, 2, 150-160.

Kolip, P. (2000): Tabak- und Alkoholkonsum bei Jugendlichen: Entwicklungstrends, Prävalenzen und Konsummuster in den alten Bundesländern. In: Leppin, A./Hurrelmann, K./Petermann, H. (Hrsg.): Jugendliche und Alltagsdrogen. Konsum und Perspektiven der Prävention. Neuwied: Luchterhand, 2444.

Kurz, D./Sack, H.-G./Brinkhoff, K.-P. (1996): Kindheit, Jugend und Sport in Nordrhein-Westfalen. Düsseldorf: Ministerium für Stadtentwicklung, Kultur und Sport.

Liebau, E. (1992): Habitus, Lebenslage und Geschlecht. Über Sozioanalyse und Geschlechtersozialisation. In: Tillmann, K.-J. (Hrsg.): Jugend weiblich - Jugend männlich. Sozialisation, Geschlecht, Identität. Opladen: Leske + Budrich, 134-148.

Lüdtke, H. (1989): Expressive Ungleichheit. Zur Soziologie der Lebensstile. Opladen: Leske + Budrich.

Lüdtke, H. (1990): Lebensstile als Dimensionen handlungsproduzierter Ungleichheit. Eine Anwendung des Rational-Choice-Ansatzes. In: Berger, P.A./Hradil, S. (Hrsg.): Lebenslagen, Lebensläufe, Lebensstile. Sonderband 7 der Sozialen Welt. Göttingen: Otto Schwarz, 433-453.

Lüdtke, H. (1992): Zwei Jugendkulturen? Freizeitmuster in Ost und West. In: Zinnecker, J. (Red.): Jugend 92. Lebenslagen, Orientierungen und Entwicklungsperspektiven im vereinigten Deutschland. Bd. 2. Opladen: Leske + Budrich, 239-264.

Markert, C. (2002): Methodische Möglichkeiten zur Abschätzung der Verlässlichkeit von Selbstaussagen in suchtpräventiven Längsschnittstudien. In: Petermann, H./Roth, M. (Hrsg.): Sucht und Suchtprävention. Berlin: Logos, 105-127.

Meyer, T. (2001): Das Konzept der Lebensstile in der Sozialstrukturforschung - eine kritische Bilanz. In: Soziale Welt, 52, 255-572. 
Michailow, M. (1994): Lebensstilsemantik. Soziale Ungleichheit und Formationsbildung in der Kulturgesellschaft. In: Mörth, I./Fröhlich, G. (Hrsg.): Das symbolische Kapital der Lebensstile. Zur Kultursoziologie der Moderne nach Pierre Bourdieu. Frankfurt: Campus, 107-128.

Mielck, A. (2000): Soziale Ungleichheit und Gesundheit. Empirische Ergebnisse, Erklärungsansätze, Interventionsmöglichkeiten. Bern: Huber.

Mitchel, A. (1983): The Nine American Life Styles. New York.

Moore, H.L. (1993): The Differences Within and the Differences Between. In: Valle, T. del (Ed.): Gendered Anthropology. London: Routledge, 193-204.

Müller, H.-P. (1992): Sozialstruktur und Lebensstile. Der neuere theoretische Diskurs über soziale Ungleichheit. Frankfurt: Suhrkamp.

Pavis, S./Cunningham-Burley, S./Amos, A. (1997): Alcohol Consumption and Young People. In: Health Education Research, 12, 3, 311-322.

Pientka, L. (1994): Gesundheitliche Ungleichheit und das Lebensstilkonzept. In: Mielck, A. (Hrsg.): Krankheit und soziale Ungleichheit. Ergebnisse der sozialepidemiologischen Forschung in Deutschland. Opladen: Leske + Budrich, 393-409.

Raithel, J. (2001): Risikoverhaltensweisen Jugendlicher - Ein Überblick. In: Raithel, J. (Hrsg.): Risikoverhaltensweisen Jugendlicher. Formen, Erklärungen und Prävention. Opladen: Leske + Budrich, 1129.

Raithel, J. (2002): Ernährungs- und Gesundheits-/Risikoverhalten Jugendlicher. In: Zeitschrift für Gesundheitswissenschaften, 10, 1, 58-71.

Raithel, J. (2003a): Risikobezogenes Verhalten und Geschlechtsrollenorientierung im Jugendalter. In: Zeitschrift für Gesundheitspsychologie, 11, 1, 21-28.

Raithel, J. (2003b): Riskante Verhaltensweisen im Jugendalter. Ein Literaturüberblick und lebensstilbezogene Forschungsperspektive. In: Zeitschrift für Soziologie der Erziehung und Sozialisation, 23, 3, 286-301.

Raithel, J. (2004): Gesundheitsrelevante Lebensstile als Modus des „,doing genders“ im jugendlichen Entwicklungsprozess. In: Zeitschrift für Soziologie der Erziehung und Sozialisation (in Druck).

Richter, R. (1989): Subtile Distinktion zur Reproduktion sozialer Ungleichheit im mikrosozialen Bereich. In: Österreichische Zeitschrift für Soziologie, 14, 3, 53-63.

Richter, R. (1994): Stile im Konflikt in der Begegnung zwischen Ost und West. Ein kultursoziologischer Beitrag aus der Lebensstilforschung. In: Schwenk, O.G. (Hrsg.): Lebensstil zwischen Sozialstrukturanalyse und Kulturwissenschaft. Opladen: Leske + Budrich, 261-282.

Schmidt, B. (1998): Suchtprävention bei konsumierenden Jugendlichen. Sekundärpräventive Ansätze in der geschlechtsbezogenen Drogenarbeit. Weinheim: Juventa.

Schwarzer, R. (1990): Gesundheitspsychologie: Einführung in das Thema. In: Schwarzer, R. (Hrsg.): Gesundheitspsychologie. Ein Lehrbuch. Göttingen: Hogrefe, 3-23.

Schulze, G. (1992): Die Erlebnisgesellschaft. Kultursoziologie der Gegenwart. Frankfurt: Campus.

Siegrist, J. (1989): Steps Towards Explaining Social Differentials in Morbidity: The Case of West Germany. In: Fox, J. (Ed.): Health Inequalities in European Countries. London: Gower Public, 353-371.

Sieverding, M. (1997): Die Bedeutung von Prototype-Matching für präventives Verhalten. In: Zeitschrift für Gesundheitspsychologie, 5, 4, 272-289.

Sieverding, M. (2000): Risikoverhalten und präventives Verhalten im Geschlechtervergleich: Ein Überblick. In: Zeitschrift für Medizinische Psychologie, 1, 7-16.

Steinkamp, G. (1993): Soziale Ungleichheit, Erkrankungsrisiko und Lebenserwartung: Kritik der sozialepidemiologischen Ungleichheitsforschung. In: Sozial- und Präventivmedizin, 38, 111-122.

Steinkamp, G. (1999): Soziale Ungleichheit in Mortalität und Morbidität: Oder: Warum einige Menschen gesünder sind und länger leben als andere. In: Schlicht, W./Dickhuth, H. (Hrsg.): Gesundheit für alle. Schorndorf: Hofmann, 101-154. 
Spellerberg, A. (1996): Soziale Differenzierung durch Lebensstile. Eine empirische Untersuchung zur Lebensqualität in West- und Ostdeutschland. Berlin: Ed. Sigma.

Sperlich, S./Mielck, A. (2003): Sozialepidemiologische Erklärungsansätze im Spannungsfeld zwischen Schicht- und Lebensstilkonzeptionen. Plädoyer für eine integrative Betrachtung auf der Grundlage der Bourdieuschen Habitustheorie. In: Zeitschrift für Gesundheitswissenschaften, 11, 2, 165-179.

StBA - Statistisches Bundesamt (2002): Gesundheitswesen. VIII A 1. Wiesbaden: Metzler Poeschel.

Tomlinson, M. (2003): Lifestyle and Social Class. In: European Sociological Review, 19, 97-111.

Ulbrich-Herrmann, M. (1998): Lebensstile Jugendlicher und Gewalt. Eine Typologie zur mehrdimensionalen Erklärung eines sozialen Problems. Münster: Lit.

Vester, M./Gardemin, D. (2000): Milieu, Klasse und Geschlecht. Das Feld der Geschlechterungleichheit und die ,protestantische Alltagsethik“. In: Heintz, B. (Hrsg.): Geschlechter-Soziologie. (Sonderheft KZfSS) Opladen: Westdeutscher, 454-486.

Ward, J.H. (1963): Hierarchical Grouping to Optimize an Objective Function. In: Journal of the American Statistical Association, 58, 244-263.

Weber, I. (1987): Soziale Schichtung und Gesundheit. In: Geißler, R. (Hrsg.): Soziale Schichtung und Lebenschancen in der Bundesrepublik. Stuttgart: Enke, 172-182.

Weber, M. (1972): Gesammelte Aufsätze zur Religionssoziologie. Bd. 1. Tübingen: Mohr (Paul Siebeck). WHO - World Health Organisation (1948): Constitution. Genf: WHO.

Ziegelmann, J.P. (2002): Gesundheits- und Risikoverhalten. In: Schwarzer, R./Jerusalem, M./Weber, H. (Hrsg.): Gesundheitspsychologie von A bis Z. Göttingen: Hogrefe, 152-155.

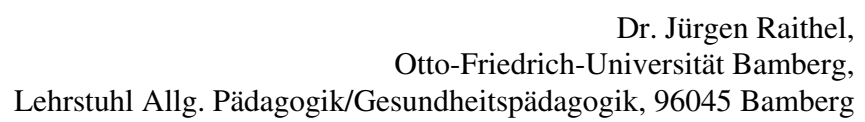
Tel: 0951-8631832, JRaithel@jraithel.de 